\title{
Iterative learning control of a distributed heating system described by a non-minimum phase model
}

\author{
Slawomir Mandra, Krzysztof Galkowski, Eric Rogers, Harald Aschemann and Andreas Rauh
}

\begin{abstract}
This paper considers iterative learning control design for non-minimum phase dynamics using a model derived from an experimental spatially distributed system, i.e., a heating system. A novel design based on an $\mathcal{H}_{\infty}$ setting and convex optimization with validation in both simulation and experiment.
\end{abstract}

\section{INTRODUCTION}

Iterative Learning Control (ILC) is a technique that allows precision tracking of a reference signal in systems that perform the same finite duration task over and over again. High performance are preserved in the presence of plant model uncertainty and unknown but repetitive disturbances. In the literature each execution of task is commonly termed a trial and the trial length is the time taken to complete a trial. The tracking error is reduced from trial-to-trial by update a control signal computed using data from the previous trial.

The first work on ILC is widely credited to [1] and since then ILC has remained an area of control systems research and implementation, where in the latter case this has extended from laboratory experiments to actual implementation. Survey papers such as [2], [3] are possible starting points for the early literature. Recent examples of the many experimental validations include [4], [5].

The ILC design for non-minimum phase system poses extra challenges compared to minimum phase plant model because a direct inversion of the system is unstable which leads to undesirably large ILC control signals [2], [6]. The results in this area applied to an electro-mechanical system with a finite-dimensional system for experimental verification are given in [7] but such a behaviour also arises in the area of spatially distributed systems. This paper reports new results on ILC design for a distributed heating system pre-stabilized by state feedback controller with a state and disturbance observer in order to obtain a small tracking error on the

This work is partially supported by National Science Centre in Poland, grant No. 2015/17/B/ST7/03703.

Slawomir Mandra (on sabbatical leave) is with the Institute of Physics, Faculty of Physics, Astronomy and Informatics, Nicolaus Copernicus University, Grudziadzka 5, 87-100 Torun, Poland manslawefizyka.umk.pl

Krzysztof Galkowski and Slawomir Mandra (postdoc) are with the Institute of Control and Computation Engineering, University of Zielona Gora, Szafrana 2, 65-516 Zielona Gora, Poland K.Galkowski@issi.uz.zgora.pl

Eric Rogers is with the Department of Electronics and Computer Science, University of Southampton, Southampton SO17 1BJ, UK etardecs.soton.ac.uk

Harald Aschemann and Andreas Rauh are with the Chair of Mechatronics, University of Rostock, Justus-von-Liebig-Weg 6, D-18059 Rostock, Germany \{harald.aschemann, andreas.rauh\} @uni-rostock. de

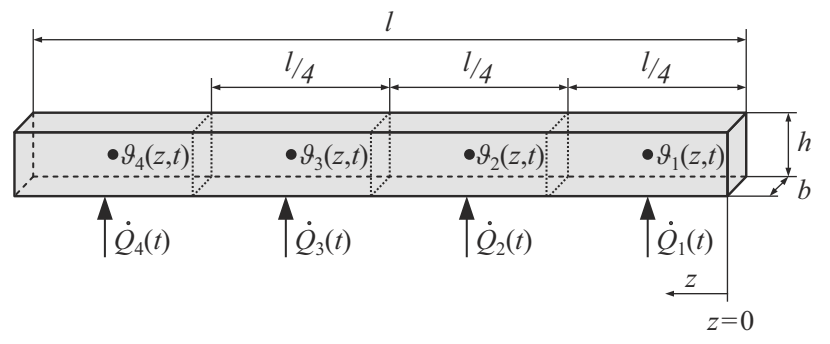

Fig. 1. Schematic representation of the heat transfer problem in a metal rod showing the actuator and sensor locations.

first trial. The considered heating system is approximated by a non-minimum phase model given in form a state-space model.

The ILC design for a non-minimum phase systems can be effectively solved in frequency domain using $\mathcal{H}_{\infty}$ method and convex optimization. But this approach commonly lead to very large magnitude of the learning filter at high frequencies which can make the ILC system sensitive on, e.g., measurement noise, uncertainty in the plant model and the initial conditions. In [8], [9] this problem is solved by introduction of the weighting function defined in frequency domain. However, this approach can cause difficulties because there are no guidelines on how to shape this function. To address this issue direct limitation of the learning filter magnitude, by specifying its maximum allowable value, is included in the constraints placed on the convex optimization. The increase of the ILC convergence speed for the selected frequency is obtained by adding to the cost function the monotonic convergence condition for the adopted frequency.

In the remainder of the paper the identity matrix with compatible dimension is denoted by $\boldsymbol{I}$ and the spectral radius of a square matrix $\boldsymbol{A}$ is denoted by $\rho(\boldsymbol{A})$. A symmetric positive semi-definite matrix is denoted by $\succeq 0$.

\section{CONTROL-ORIENTED MODELLING OF THE DISTRIBUTED HEATING SYSTEM}

Fig. 1 shows a schematic view of a metal rod that can be heated or cooled from the bottom by four independent Peltier elements, i.e., actuators, generating heat flows $\dot{Q}_{i}(t), i=1, \ldots, 4$. All of the side surfaces of a rod are thermally insulated and the top surface is in direct contact with the atmosphere and temperature $\vartheta_{\mathrm{a}}(t)$. It is assumed that measurements $\vartheta_{i}(z, t)$ can be made at the geometric midpoints of the four segments of the rod. The length $l$ of the rod is much greater than the height $h$ and the width $b$. This paper is based on an experimental facility [10] that has four 
actuators but the analysis holds with routine modifications for any finite number of segments heated by Peltier elements.

Under the assumptions made, a spatial one-dimensional temperature distribution $\vartheta(z, t)$ can be assumed and modeled by an application of the first law of thermodynamics as [10]

$$
\frac{\partial q(z, t)}{\partial z}+\kappa_{1} \frac{\partial \vartheta(z, t)}{\partial t}+\kappa_{2} \vartheta(z, t)=\mu_{\mathrm{c}}(z, t)+\mu_{\mathrm{d}}(t)
$$

and by application of Fourier's heat conduction law

$$
q(z, t)=-\lambda \frac{\partial \vartheta(z, t)}{\partial z}
$$

where $q(z, t)$ is the heat flux density, the coefficients $\kappa_{1}=$ $\rho c_{p}$ and $\kappa_{2}=\frac{\alpha}{h}$ depend on the density $\rho$, the specific heat capacity $c_{p}$, the convective heat transfer coefficient $\alpha$, the height $h$ of the rod and $\lambda$ is the heat conductivity. The control input $\mu_{\mathrm{c}}(z, t)$ is generated by the Peltier elements and described by

$$
\mu_{\mathrm{c}}(z, t)=\sum_{i=1}^{4} a_{\mathrm{c}, i}(z) \dot{Q}_{i}(t)
$$

where

$$
a_{\mathrm{c}, i}(z)= \begin{cases}\frac{4}{b h l} & \text { for } z \in\left[z_{i-1}, z_{i}\right] \\ 0 & \text { otherwise. }\end{cases}
$$

The positions $z_{i}=i \frac{l}{4}, i=1,2,3,4$, along the $z$-axis in Fig. 1 are the edges of the Peltier elements. The spatially invariant disturbance $\mu_{\mathrm{d}}(t)$ caused by the time-varying ambient temperature is represented by

$$
\mu_{\mathrm{d}}(t)=\kappa_{2} \vartheta_{\mathrm{a}}(t)
$$

and the boundary conditions for the heat flux density $q(z, t)$ are assumed to be $q(0, t)=\bar{q}_{0}(t)$ and $q(l, t)=\bar{q}_{l}(t)$, where $\bar{q}_{0}(t)=0$ and $\bar{q}_{l}(t)=0$ since the side surfaces are isolated. The initial temperature distribution in the rod is taken as $\vartheta(z, 0)=\bar{\vartheta}_{0}(z)$.

Substituting (2) into (1) gives the parabolic partial differential equation that describes the spatially distributed temperature in the rod. In this paper, control system design is based on approximating the dynamics by a set of ordinary differential equations (ODEs). To construct this approximation, the method of integro-differential relations (MIDR) combined with the projection approach is used [10]. In this method, the temperature profile in the rod is approximated by

$$
\tilde{\vartheta}(z, t)=\sum_{i=1}^{4} \sum_{m=0}^{M} b_{i, m, M}(z) \theta_{i, m, M}(t)
$$

where $\theta_{i, m, M}$ are the unknown time-dependent coefficients and $b_{i, m, M}(z)$ are Bernstein polynomials of degree $M$. These polynomials guarantee continuity of the temperature distribution between neighbouring segments $i$ and $i+1$ defined by the edges of the Peltier elements

$$
\begin{aligned}
b_{i, m, M}(z) & = \begin{cases}b_{i}^{m, M}(z) & \text { for } z \in\left[z_{i-1}, z_{i}\right] \\
0 & \text { otherwise, }\end{cases} \\
b_{i}^{m, M}(z) & =\left(\begin{array}{c}
M \\
m
\end{array}\right)\left(\frac{z-z_{i-1}}{z_{i}-z_{i-1}}\right)^{m}\left(\frac{z_{i}-z}{z_{i}-z_{i-1}}\right)^{M-m} .
\end{aligned}
$$

Moreover, by inter-segment temperature continuity,

$$
\theta_{i, M, M}(t)=\theta_{i+1,0, M}(t) .
$$

The heating system model in form of ODEs is derived by solving [10]

$$
\int_{z_{i-1}}^{z_{i}}\left(\xi(z, t, \tilde{\vartheta}) \cdot b_{i, m, M-1}(z)\right) \mathrm{d} z=0
$$

for each segment $i=1, \ldots, 4$ and each polynomial degree $m \in\{0, \ldots, M-1\}$ and

$$
\begin{aligned}
\int_{0}^{l}\left[\kappa_{1} \frac{\partial \tilde{\vartheta}(x, t)}{\partial t}+\right. & \left.\kappa_{2} \tilde{\vartheta}(x, t)\right] \mathrm{d} x \\
& =\int_{0}^{l} \mu(x, t) \mathrm{d} x+\bar{q}_{0}(t)-\bar{q}_{l}(t),
\end{aligned}
$$

where

$$
\begin{aligned}
\xi(z, t, \tilde{\vartheta}):= & \int_{0}^{z}\left[\mu(x, t)-\kappa_{1} \frac{\partial \tilde{\vartheta}(x, t)}{\partial t}-\kappa_{2} \tilde{\vartheta}(x, t)\right] \mathrm{d} x \\
& +\lambda \frac{\partial \tilde{\vartheta}(z, t)}{\partial z}+\bar{q}_{0}(t)=0 \\
& \mu(x, t)=\mu_{\mathrm{c}}(x, t)+\mu_{\mathrm{d}}(t)
\end{aligned}
$$

\section{CONTROL CONFIGURATION}

The control problem is to track the reference temperature at the geometric midpoint of the second segment with control applied only through the first Peltier element in Fig. 1. In the nominal case, all other heating elements are turned off. Disturbances result from changes in the ambient temperature together with actuation of the other heating elements which is however unknown to the controller. The measurable variable is only the temperature in the geometric midpoint of the second segment of the rod. In the remainder of this paper all state variables are relative to the initial ambient temperature.

The linear continuous-time state-space model of heating system obtained by solving (9) and (10) for particular case of $M=3$ (selected as one of many choices) is given by

$$
\begin{aligned}
\dot{\boldsymbol{x}}(t) & =\boldsymbol{A}_{\mathrm{c}} \boldsymbol{x}(t)+\boldsymbol{B}_{\mathrm{c}} u(t)+\boldsymbol{E}_{\mathrm{c}} d(t), \\
y(t) & =\boldsymbol{C}_{\mathrm{c}} \boldsymbol{x}(t),
\end{aligned}
$$

with the state vector $\boldsymbol{x}(t)=\left[\theta_{1,0,3}(t) \theta_{1,1,3}(t) \ldots\right.$ $\left.\theta_{4,3,3}(t)\right]^{\mathrm{T}} \in \mathbb{R}^{13}$ consisting of the Bernstein coefficients, the control input $u(t)=\dot{Q}_{1}(t)$, the disturbance input $d(t)=$ $\vartheta_{\mathrm{a}}(t)$ due to changes in the ambient temperature and the output $y(t)=\vartheta_{2}(t)$. The model has been generated using the following data: $l=0.320 \mathrm{~m}, b=0.040 \mathrm{~m}, h=0.012 \mathrm{~m}$, $\rho=7800 \mathrm{~kg} / \mathrm{m}^{3}, c_{p}=420 \mathrm{~J} /(\mathrm{kg} \cdot \mathrm{K}), \alpha=150 \mathrm{~W} /\left(\mathrm{m}^{2} \cdot \mathrm{K}\right)$ and $\lambda=55 \mathrm{~W} /(\mathrm{m} \cdot \mathrm{K})$. 
A minimal realisation was constructed by canceling three stable pole-zero pairs. The resulting model transfer-function has nine zeros, one of which is in the right-hand half of the $s$-plane (at $s=0.1011$ ) and all poles are stable. For ILC design, this model has been discretized using exact method with sampling period $T_{\mathrm{s}}=1 \mathrm{~s}$ and written in the ILC setting on trial $k+1$ as

$$
\begin{aligned}
\boldsymbol{x}_{k+1}(p+1) & =\boldsymbol{A} \boldsymbol{x}_{k+1}(p)+\boldsymbol{B} u_{k+1}(p)+\boldsymbol{E} d_{k+1}(p), \\
y_{k+1}(p) & =\boldsymbol{C} \boldsymbol{x}_{k+1}(p) .
\end{aligned}
$$

where $\boldsymbol{x}_{k+1}(p) \in \mathbb{R}^{10}$. The discrete-time plant model (14) is also non-minimum phase and has the relative degree $r=1$.

The developed ILC scheme for the distributed heating system is shown in Fig. 2. It is based on a state feedback controller, a state and disturbance Luenberger observer and a static feedforward controller to result in a small tracking error starting from the first trial at which the ILC signal $v_{1}(p)=0$.

For disturbance observer design and disturbance compensation purposes, the plant model (14) can be rewritten in the following equivalent form where the disturbance only acts on the plant input

$$
\begin{aligned}
\boldsymbol{x}_{k+1}(p+1) & =\boldsymbol{A} \boldsymbol{x}_{k+1}(p)+\boldsymbol{B}\left(u_{k+1}(p)+\delta_{k+1}(p)\right), \\
y_{k+1}(p) & =\boldsymbol{C} \boldsymbol{x}_{k+1}(p),
\end{aligned}
$$

where $\delta_{k+1}(p)$ is a lumped, additive input disturbance [11] equivalent to variations of both the ambient temperature and the non-modelled external disturbance heat flows acting on the metallic rod. The control law of the internal control loop shown in Fig. 2 is given by

$$
u_{k+1}(p)=N w_{k+1}(p)-\boldsymbol{K}_{\mathrm{s}} \hat{\boldsymbol{x}}_{k+1}(p)-\hat{\delta}_{k+1}(p),
$$

where $N$ is the static feedforward gain, $w_{k+1}(p)$ is the input to the state feedback control system, $\boldsymbol{K}_{\mathrm{s}}$ is the feedback gain vector, $\hat{\boldsymbol{x}}_{k+1}(p)$ is the estimate of $\boldsymbol{x}_{k+1}(p)$ and $\hat{\delta}_{k+1}(p)$ is the estimate of $\delta_{k+1}(p)$. For constant or slowly varying disturbance, the commonly used disturbance model is in the form of the integral action

$$
\begin{aligned}
\varepsilon_{k+1}(p+1) & =A_{\mathrm{d}} \varepsilon_{k+1}(p), \\
\delta_{k+1}(p) & =C_{\mathrm{d}} \varepsilon_{k+1}(p),
\end{aligned}
$$

where both $A_{\mathrm{d}}=C_{\mathrm{d}}=1$.

The plant model (15) augmented with disturbance model (17) is given by

$$
\begin{aligned}
\boldsymbol{z}_{k+1}(p+1) & =\boldsymbol{A}_{\mathrm{a}} \boldsymbol{z}_{k+1}(p)+\boldsymbol{B}_{\mathrm{a}} u_{k+1}(p), \\
y(p) & =\boldsymbol{C}_{\mathrm{a}} \boldsymbol{z}_{k+1}(p),
\end{aligned}
$$

where

$$
\begin{aligned}
\boldsymbol{A}_{\mathrm{a}} & =\left[\begin{array}{cc}
\boldsymbol{A} & \boldsymbol{B}_{C_{\mathrm{d}}} \\
\mathbf{0} & A_{\mathrm{d}}
\end{array}\right], \quad \boldsymbol{z}_{k+1}(p)=\left[\begin{array}{l}
\boldsymbol{x}_{k+1}(p) \\
\varepsilon_{k+1}(p)
\end{array}\right], \\
\boldsymbol{B}_{\mathrm{a}} & =\left[\begin{array}{c}
\boldsymbol{B} \\
0
\end{array}\right], \quad \boldsymbol{C}_{\mathrm{a}}=\left[\begin{array}{ll}
\boldsymbol{C} & 0
\end{array}\right] .
\end{aligned}
$$

Then, the estimates of the state vector and the equivalent disturbance $\delta_{k+1}(p)$ can be obtained using the Luenberger

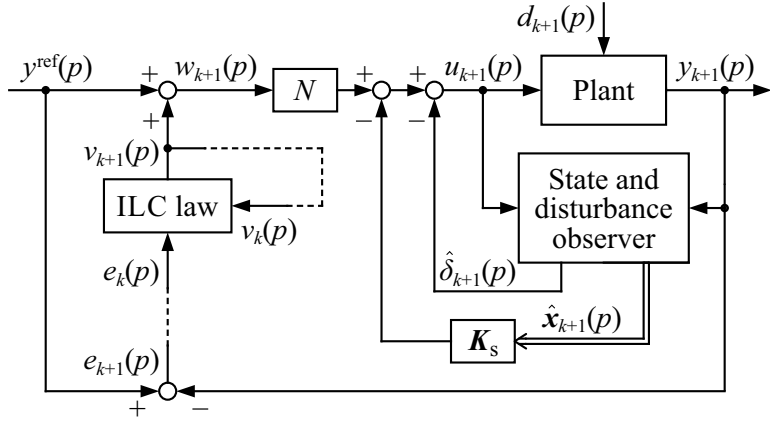

Fig. 2. Block diagram of the ILC structure based on a state feedback control law, a Luenberger state and disturbance observer and the static feedforward gain $N$.

observer

$$
\begin{aligned}
\hat{\boldsymbol{z}}_{k+1}(p+1)= & \boldsymbol{A}_{\mathrm{a}} \hat{\boldsymbol{z}}_{k+1}(p)+\boldsymbol{B}_{\mathrm{a}} u_{k+1}(p) \\
& +\boldsymbol{K}_{\mathrm{L}}\left(y_{k+1}(p)-\boldsymbol{C}_{\mathrm{a}} \hat{\boldsymbol{z}}_{k+1}(p)\right),
\end{aligned}
$$

where $\hat{\boldsymbol{z}}_{k+1}(p)=\left[\begin{array}{ll}\hat{\boldsymbol{x}}_{k+1}^{\mathrm{T}}(p) & \hat{\varepsilon}_{k+1}(p)\end{array}\right]^{\mathrm{T}}$ is the observer state vector and $\boldsymbol{K}_{\mathrm{L}}=\left[\begin{array}{ll}\boldsymbol{K}_{\mathrm{L} 1}^{\mathrm{T}} & K_{\mathrm{L} 2}\end{array}\right]^{\mathrm{T}}$ is the observer gain vector. The state-space model of the internal control loop is given by

$$
\begin{aligned}
\chi_{k+1}(p+1) & =\boldsymbol{A}_{\mathrm{cl}} \chi_{k+1}(p)+\boldsymbol{B}_{\mathrm{cl}} w_{k+1}(p)+\boldsymbol{E}_{\mathrm{cl}} d_{k+1}(p), \\
y_{k+1}(p) & =\boldsymbol{C}_{\mathrm{cl}} \chi_{k+1}(p),
\end{aligned}
$$

where

$$
\begin{aligned}
\boldsymbol{A}_{\mathrm{cl}} & =\left[\begin{array}{ccc}
\boldsymbol{A} & -\boldsymbol{B} \boldsymbol{K}_{\mathrm{s}} & -\boldsymbol{B} C_{\mathrm{d}} \\
\boldsymbol{K}_{\mathrm{L} 1} \boldsymbol{C} & \boldsymbol{A}-\boldsymbol{B} \boldsymbol{K}_{\mathrm{s}}-\boldsymbol{K}_{\mathrm{L} 1} \boldsymbol{C} & \mathbf{0} \\
K_{\mathrm{L} 2} \boldsymbol{C} & -K_{\mathrm{L} 2} \boldsymbol{C} & A_{\mathrm{d}}
\end{array}\right], \\
\boldsymbol{B}_{\mathrm{cl}} & =\left[\begin{array}{c}
\boldsymbol{B} N \\
\boldsymbol{B} N \\
0
\end{array}\right], \\
\boldsymbol{E}_{\mathrm{cl}} & =\left[\begin{array}{lll}
\boldsymbol{C} & \mathbf{0} & 0
\end{array}\right] .\left[\begin{array}{l}
\boldsymbol{E} \\
\mathbf{0} \\
0
\end{array}\right], \quad \boldsymbol{\chi}_{k+1}(p)=\left[\begin{array}{l}
\boldsymbol{x}_{k+1}(p) \\
\hat{\boldsymbol{x}}_{k+1}(p) \\
\hat{\varepsilon}_{k+1}(p)
\end{array}\right],
\end{aligned}
$$

The aim of the ILC design is improve tracking performance by adding to the reference signal $\left(y^{\mathrm{ref}}(p)\right)$ the ILC signal $v_{k+1}(p)$

$$
w_{k+1}(p)=y^{\mathrm{ref}}(p)+v_{k+1}(p)
$$

such that the tracking error

$$
e_{k+1}(p)=y^{\mathrm{ref}}(p)-y_{k+1}(p)
$$

not compensated for by the internal control loop with dynamics (21) is reduced over subsequent trials. For this purpose, the ILC law, see, e.g. [2]

$$
v_{k+1}(p)=Q(q)\left(v_{k}(p)+L(q) e_{k}(p)\right)
$$

is used where $Q(q)$ is a zero-phase, low-pass filter, $L(q)$ is the learning filter and $q$ is the forward time-shift operator $q e_{k}(p) \equiv e_{k}(p+1)$. In this paper it is assumed that Q-filter is low-pass, Butterworth filter of order $n$ applied forward and backward to the signal, resulting in a $2 n$-th order zero-phase 
low-pass filter written as

$$
\begin{aligned}
Q(q)= & \frac{b_{0}+b_{1} q^{-1}+b_{2} q^{-2}+\cdots+b_{n} q^{-n}}{1+a_{1} q^{-1}+a_{2} q^{-2}+\cdots+a_{n} q^{-n}} \\
& \cdot \frac{b_{0}+b_{1} q+b_{2} q^{2} \cdots+b_{n} q^{n}}{1+a_{1} q+a_{2} q^{2}+\cdots+a_{n} q^{n}} .
\end{aligned}
$$

The learning filter has the form of a finite impulse response filter

$$
L(q)=l_{1} q+l_{2} q^{2}+\cdots+l_{s} q^{s} .
$$

In the next section the design of both the internal control system and the ILC law are described.

\section{CONTROL SYSTEM DESIGN}

The standard conditions for monotonic tracking error convergence of ILC algorithm can be applied to considered system and lead to [2]

i) $\rho\left(\boldsymbol{A}_{\mathrm{cl}}\right)<1$

ii) $\left\|Q(q)\left(1-P_{\mathrm{wy}}(q) L(q)\right)\right\|_{\infty}<1, \quad \forall q \in \mathcal{D}$

where

$$
\begin{aligned}
& P_{\mathrm{wy}}(q)=\boldsymbol{C}_{\mathrm{cl}}\left(q \boldsymbol{I}-\boldsymbol{A}_{\mathrm{cl}}\right)^{-1} \boldsymbol{B}_{\mathrm{cl}}, \\
& \mathcal{D}=\left\{q=e^{j \omega T_{\mathrm{s}}} \mid \omega \in\left[0, \omega_{\mathrm{N}}\right]\right\},
\end{aligned}
$$

$\omega_{\mathrm{N}}$ is the Nyquist angular frequency.

The first condition requires that $\boldsymbol{A}_{\mathrm{cl}}$ is a stable matrix in the standard linear systems sense. In this case, the separation principle of linear feedback control and observer design can be used. To design the state feedback matrix $\boldsymbol{K}_{\mathrm{s}}$ many possible algorithms exist and in this paper the design is completed by minimizing the linear quadratic cost function

$$
J_{\mathrm{s}}=\sum_{p=0}^{\infty}\left(\boldsymbol{x}_{k+1}^{\mathrm{T}}(p) \boldsymbol{Q}_{\mathrm{s}} \boldsymbol{x}_{k+1}(p)+R_{\mathrm{s}} u_{k+1}^{2}(p)\right),
$$

where $\boldsymbol{Q}_{\mathrm{s}} \succeq 0$ and $R_{\mathrm{s}}>0$ are an appropriately chosen weighting matrix and scalar, respectively. This optimization problem leads to the discrete-time matrix Riccati equation

$$
\boldsymbol{A}^{\mathrm{T}} \boldsymbol{P}_{\mathrm{s}}\left(\boldsymbol{I}-\boldsymbol{B}\left(\boldsymbol{B}^{\mathrm{T}} \boldsymbol{P}_{\mathrm{s}} \boldsymbol{B}+R_{\mathrm{s}}\right)^{-1} \boldsymbol{B}^{\mathrm{T}} \boldsymbol{P}_{\mathrm{s}}\right) \boldsymbol{A}+\boldsymbol{Q}_{\mathrm{s}}=\boldsymbol{P}_{\mathrm{s}}
$$

The solution matrix $\boldsymbol{P}_{\mathrm{S}}$ of this last equation is used to compute $\boldsymbol{K}_{\mathrm{s}}$ as

$$
\boldsymbol{K}_{\mathrm{s}}=\left(\boldsymbol{B}^{\mathrm{T}} \boldsymbol{P}_{\mathrm{s}} \boldsymbol{B}+R_{\mathrm{s}}\right)^{-1}\left(\boldsymbol{B}^{\mathrm{T}} \boldsymbol{P}_{\mathrm{s}} \boldsymbol{A}\right) .
$$

To design the observer gain vector $\boldsymbol{K}_{\mathrm{L}}$, the following quadratic cost function is used

$$
J_{\mathrm{o}}=\sum_{p=0}^{\infty}\left(\breve{\boldsymbol{z}}_{k+1}^{\mathrm{T}}(p) \boldsymbol{Q}_{\mathrm{o}} \breve{\boldsymbol{z}}_{k+1}(p)+R_{\mathrm{o}} \breve{y}_{k+1}^{2}(p)\right),
$$

where $\breve{\boldsymbol{z}}_{k+1}(p)=\boldsymbol{z}_{k+1}(p)-\hat{\boldsymbol{z}}_{k+1}(p)$ is the state estimation error, $\breve{y}_{k+1}(p)=y_{k+1}(p)-\hat{y}_{k+1}(p)$ the output estimation error, $\boldsymbol{Q}_{\mathrm{o}} \succeq 0$ and the scalar $R_{\mathrm{o}}>0$ are an appropriately chosen weighting matrix and scalar, respectively. The solution matrix $\boldsymbol{P}_{\mathrm{O}}$ of the following discrete-time matrix Riccati equation

$$
\boldsymbol{A} \boldsymbol{P}_{\mathrm{o}}\left(\boldsymbol{I}-\boldsymbol{C}^{\mathrm{T}}\left(\boldsymbol{C} \boldsymbol{P}_{\mathrm{o}} \boldsymbol{C}^{\mathrm{T}}+R_{\mathrm{o}}\right)^{-1} \boldsymbol{C} \boldsymbol{P}_{\mathrm{o}}\right) \boldsymbol{A}^{\mathrm{T}}+\boldsymbol{Q}_{\mathrm{o}}=\boldsymbol{P}_{\mathrm{o}}
$$

is used to calculate the optimal observer gain vector

$$
\boldsymbol{K}_{\mathrm{L}}=\left(\left(\boldsymbol{C} \boldsymbol{P}_{\mathrm{o}} \boldsymbol{C}^{\mathrm{T}}+R_{\mathrm{o}}\right)^{-1}\left(\boldsymbol{C} \boldsymbol{P}_{\mathrm{o}} \boldsymbol{A}^{\mathrm{T}}\right)\right)^{\mathrm{T}} .
$$

The ILC design for the non-minimum phase internal control system will be made in frequency domain by solving the following convex optimization problem

$$
\begin{array}{cl}
\underset{L}{\operatorname{minimize}} & \gamma+\left|Q\left(e^{j \omega_{\mathrm{r}} T_{\mathrm{s}}}\right)\left(1-P_{\mathrm{wy}}\left(e^{j \omega_{\mathrm{r}} T_{\mathrm{s}}}\right) L\left(e^{j \omega_{\mathrm{r}} T_{\mathrm{s}}}\right)\right)\right| \\
\text { subject to } & \gamma<1, \\
& \left|Q\left(e^{j \omega_{i} T_{\mathrm{s}}}\right)\left(1-P_{\mathrm{wy}}\left(e^{j \omega_{i} T_{\mathrm{s}}}\right) L\left(e^{j \omega_{i} T_{\mathrm{s}}}\right)\right)\right| \leq \gamma, \\
& \left|L\left(e^{j \omega_{i} T_{\mathrm{s}}}\right)\right| \leq g, \quad \forall \omega_{i} \in\left[0<\omega_{1}<\ldots<\omega_{\mathrm{N}}\right] .
\end{array}
$$

for given Q-filter (26). The approach is an extension of the $\mathcal{H}_{\infty}$ method [8] where the last constraint is added to limit the maximum value of the learning filter magnitude to the chosen value $g$. In [8], [9] this problem has been solved by an introduction of a weighting function defined in frequency domain. However, the constraint imposed on the learning function can cause a decrease of the ILC convergence speed in the frequency band for which the frequency spectrum of the reference signal has many components. To avoid this problem, the cost function has been enriched by adding the second term that aims to increase a convergence rate for specified angular frequency $\omega_{\mathrm{r}}$.

Note also that the second condition of ILC stability (29) should be satisfied for every $\omega$ belonging to the interval $\left[0, \omega_{N}\right]$, i.e. for infinite number of values. This condition can be relaxed such that only a finite number of reasonable chosen angular frequency $\omega_{i}$ defined in (38) is considered. The cut-off frequency of the Q-filter can be set from the analysis of the pass band of the internal control system $P_{\text {wy }}$ of (30).

\section{CASE STUDY}

In this section the numerical values of the system model given in Section III are used. The free parameters in the optimization problems (32) and (35) have been chosen for the results given as $\boldsymbol{Q}_{\mathrm{s}}=\boldsymbol{C}^{\mathrm{T}} \boldsymbol{C}, R_{\mathrm{s}}=0.05, \boldsymbol{Q}_{\mathrm{o}}=\operatorname{diag}(\boldsymbol{I}, 2)$ and $R_{\mathrm{O}}=1$ to obtain a compromise between a high dynamic and a suppression of high frequency noise. The analysis was performed for the measurement noise with the standard deviation $0.1 \mathrm{~K}$, as many temperature sensors have such an accuracy.

Completing the design gives $\boldsymbol{K}_{\mathrm{s}}=[-0.05810 .3766$ $\begin{array}{llllllll}0.3219 & -0.3210 & 0.2562 & 0.4222 & 0.3132 & 0.2139 & -0.1572\end{array}$ $0.0329], \boldsymbol{K}_{\mathrm{L}}=\left[\begin{array}{lllll}-0.6993 & 0.8131 & 1.0187 & -0.7220 & -0.1548\end{array}\right.$ $0.4797-0.1985-0.0746-0.3859-0.10381 .0739]^{\mathrm{T}}$. The static feedforward gain $N=4.8575$ has been taken as the inverse of the DC gain of the closed-loop feedback control system to ensure a small steady state error for the first trial. The Bode diagram of the internal control loop between the input of the state feedback control system $\left(w_{k+1}\right)$ and the plant output $\left(y_{k+1}\right)$ calculated based on (30) is shown in Fig. 3.

The Q-filter (26) was parametrized as a low-pass, 2thorder Butterworth filter with cutoff frequency of $0.02 \mathrm{~Hz}$. 

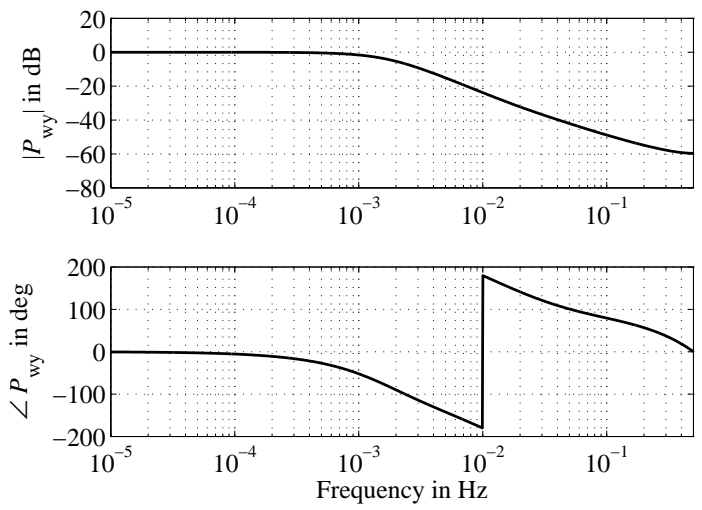

Fig. 3. Bode diagram of the internal control loop between the input of the state feedback control system and the plant output.
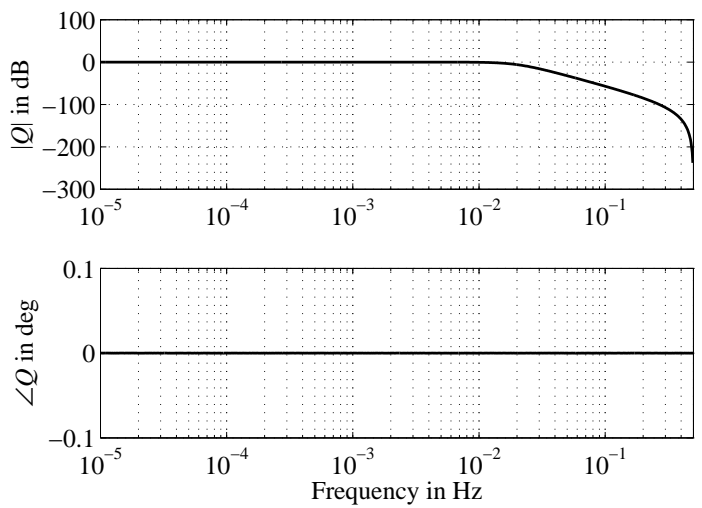

Fig. 4. Bode diagram of the $Q$-filter.

For this frequency the internal control loop significantly damps the input signal $w_{k+1}$ as the magnitude of $P_{\mathrm{wy}}$ at this frequency is approximately 0.03 . The Bode diagram of Q-filter is depicted in Fig. 4. The order of the learning filter (27) ia assumed equal to $s=10$, based on the order of the plant model (14). The internal control loop described by (21) and (30) is 21th order, where the 11 additional states are introduced by the state and disturbance observer (20).

For comparison purposes, the coefficients of $L(q)$ were calculated using i) the $\mathcal{H}_{\infty}$ method, ii) the $\mathcal{H}_{\infty}$ method with the additional condition

$$
\left|L\left(e^{j \omega_{i}}\right)\right| \leq g=20, \quad \forall \omega_{i} \in\left[0<\omega_{1}<\ldots<\omega_{\mathrm{N}}\right]
$$

limiting the magnitude of $L$ to $g=20$ and iii) the optimization procedure (38) for the same value of $g$. All optimization problems were solved using MATLAB toolboxes CVX and SDPT3 for 1000 values of $\omega_{i}$ in the range $\left[0, \omega_{\mathrm{N}}=\pi\right]$.

Fig. 5 shows the magnitude of learning filter (top) and the convergence speed of ILC system (bottom) for the learning filter coefficients calculated using the first method. For this design the $\mathcal{H}_{\infty}$ norm was 0.093 . Theoretically it can lead to very high convergence speed in wide range of frequency but due to a large learning filter magnitude at high frequency, the ILC design is sensitive to measurement noise and modelling errors.
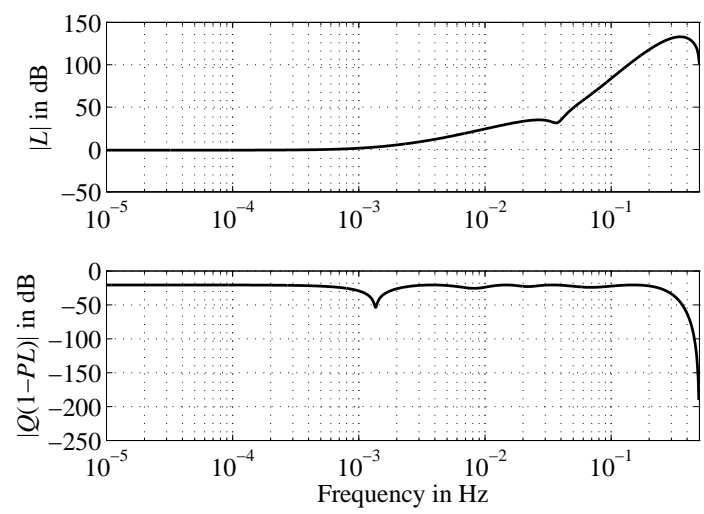

Fig. 5. Magnitude of $|L|$ and $|Q(1-P L)|$ for the $L$ coefficients calculated by minimization of $\mathcal{H}_{\infty}$ norm.

The second design of the $L$ coefficients has been undertaken using $\mathcal{H}_{\infty}$ method together with additional constraint (39) limiting magnitude of $L$ to value $g=20$. In general, the value of $g$ should be determined by user using trial-and-error method specific to the application consideration. This limitation was added to increase robustness of the ILC algorithm against the measurement noise and the uncertainty in the plant model. In this paper, a low value of $g$ has been assumed demonstrate the feasibility of (38). The $\mathcal{H}_{\infty}$ norm for the second design is 0.869 and the gray plots in Fig. 6 show the magnitude of $L$ (top) and the ILC convergence speed (bottom).

Limiting the learning filter magnitude to 20 causes a decrease of the ILC convergence speed in comparison to the previous result. To examine the performance of this design, simulations have been run over 10 trials for the control scheme of Fig. 2. Fig. 7 shows the reference signal, i.e., the desired temperature at the geometric midpoint of the second segment of the rod and the disturbance in form of the heat flow changes of the third Peltier element $\dot{Q}_{3}$ starting from the 5 th trial, where zero boundary conditions have been enforced. The output variable is the temperature in the geometric midpoint of the second segment. To compare the trial-to-trial convergence, the root-mean-square (RMS) tracking error $\operatorname{RMS}\left(e_{k}\right)$ along each trial is used and the gray plot in Fig. 8 shows the data generated for the second design.

The third design of the $L$ coefficients were completed using the optimization procedure (38), where the second component of the cost function was calculated for $\omega_{\mathrm{r}}=0$ because the frequency spectrum of the reference signal shown in Fig. 7 has many components for low frequencies. In this case, the $\mathcal{H}_{\infty}$ norm was 0.931 . The black dashed plots in Fig. 6 show the magnitude of $L$ (top) and the ILC convergence speed (bottom). The increase in convergence speed for low frequencies compared to the previous design is significant despite the identical limitation of the maximum value of the learning filter magnitude. An identical set of simulations as for the previous design were undertaken. The resulting RMS tracking error is given in Fig. 8 by the black dashed plot. The result is faster ILC convergence 

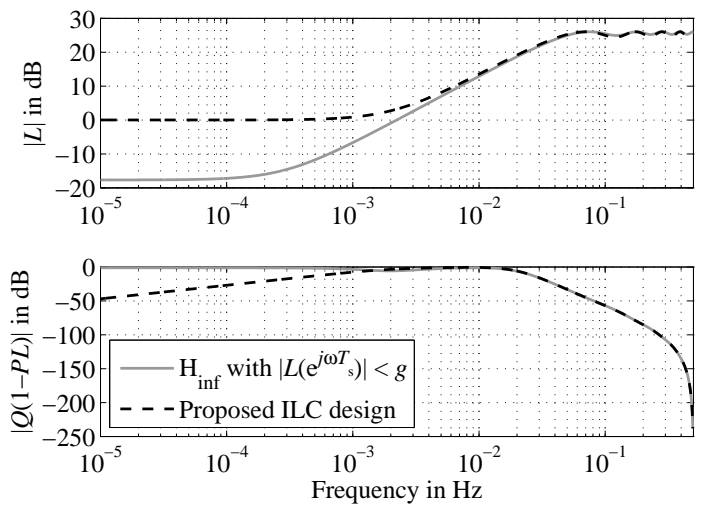

Fig. 6. Magnitude of $|L|$ and $|Q(1-P L)|$ for the $L$ coefficients calculated by minimization of $\mathcal{H}_{\infty}$ norm with additional condition (39) (gray plot) and by solving the proposed optimization problem (38) (the black dashed plot).
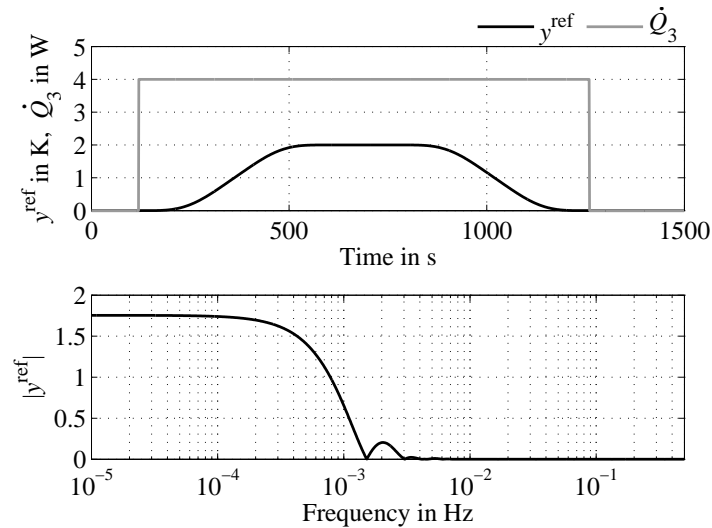

Fig. 7. The reference and disturbance signals (top). Frequency spectrum of the reference signal (bottom).

for frequency spectrum of the reference signal although the maximum magnitude of $|L|$ is still $\leq 20$.

Finally, experimental validation of the last $L$ design has been undertaken. The RMS tracking error for this design is shown in Fig. 8 using black solid line and good agreement between simulation and experiment is evident. The experimental results also indicate that the control system is robust against measurement noise, plant model uncertainty, changes of ambient temperature (during experiment the temperature in the laboratory increased by about $3 \mathrm{~K}$ ) and changes of temperatures in rod caused by heat flow generated by the third Peltier element. The ILC design needs 3 trials to obtain an RMS error below $0.1 \mathrm{~K}$. Slow disturbances are compensated on the current trial by the disturbance observer.

\section{CONCLUSIONS AND FUTURE WORKS}

In this paper, an ILC design has been developed and verified by numerical simulations and experimental research on spatially distributed heating system. For design purposes, the experimental setup is approximated by a non-minimum phase model in form of a state-space model. The new ILC design is in an $\mathcal{H}_{\infty}$ method and convex optimization where additional constraints were added to limit the maximum

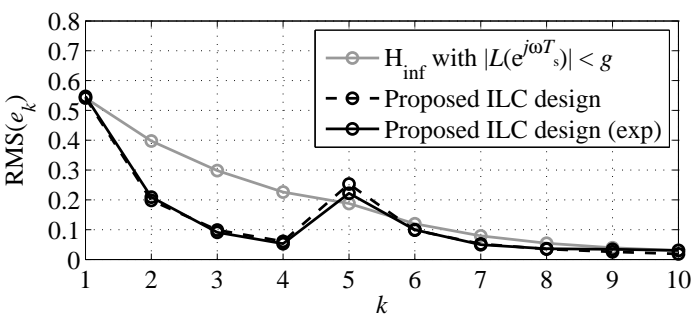

Fig. 8. RMS values of the tracking error.

value of the learning filter magnitude. The increase in the ILC convergence speed in the frequency band for which the frequency spectrum of the reference signal has many components has been obtained by adding to the cost function a second component that is the convergence condition for the selected angular frequency $\omega_{\mathrm{r}}$. The simulation and experimental results confirm that the new design is capable of high control performance. Future works will concentrate on ILC design for multi-input multi-output of distributed heating systems and experimental validation of the resulting designs.

\section{REFERENCES}

[1] S. Arimoto, S. Kawamura, F. Miyazaki, Bettering operation of robots by learning, Journal of Robotic Systems 1 (2) (1984) 123-140. doi:10.1002/rob.4620010203.

[2] D. A. Bristow, M. Tharayil, A. G. Alleyne, A survey of iterative learning control, IEEE Control Systems Magazine 26 (3) (2006) 96114. doi:10.1109/MCS.2006.1636313.

[3] H.-S. Ahn, Y.-Q. Chen, K. L. Moore, Iterative learning control: Brief survey and categorization, IEEE Transactions on Systems, Man and Cybernetics, Part C 37 (6) (2007) 1099-1121. doi:10.1109/TSMCC.2007.905759.

[4] O. Sörnmo, B. Bernhardsson, O. Kröling, P. Gunnarsson, R. Tenghamn, Frequency-domain iterative learning control of a marine vibrator, Control Engineering Practice 47 (2016) 70-80. doi:10.1016/j.conengprac.2015.12.014.

[5] S. Mandra, K. Galkowski, H. Aschemann, Robust guaranteed cost ILC with dynamic feedforward and disturbance compensation for accurate PMSM position control, Control Engineering Practice 65 (2017) 36 47. doi:10.1016/j.conengprac.2017.05.004.

[6] J. van Zundert, T. Oomen, Inverting nonminimum-phase systems from the perspectives of feedforward and ILC, in: The 20th World Congress of the International Federation of Automatic Control (IFAC), 2017, pp. $12607-12612$

[7] D. H. Owens, B. Chu, E. Rogers, C. T. Freeman, P. L. Lewin, Influence of nonminimum phase zeros on the performance of optimal continuous-time iterative learning control, IEEE Transactions on Control Systems Technology 22 (3) (2014) 1151-1158. doi:10.1109/TCST.2013.2267743.

[8] K. Inaba, C.-C. Wang, M. Tomizuka, A. Packard, Design of iterative learning controller based on frequency domain linear matrix inequality, in: 2009 American Control Conference, 2009, pp. 246251. doi:10.1109/ACC.2009.5160710,

[9] T. D. Son, G. Pipeleers, J. Swevers, Multi-objective iterative learning control using convex optimization, European Journal of Control 33 (2017) 35 - 42. doi:10.1016/j.ejcon.2016.10.001.

[10] A. Rauh, L. Senkel, H. Aschemann, V. V. Saurin, G. V. Kostin, An integrodifferential approach to modeling, control, state estimation and optimization for heat transfer systems, International Journal of Applied Mathematics and Computer Science 26 (2016) 15-30. doi:10.1515/amcs-2016-0002

[11] J.-H. She, H. Kobayashi, Y. Ohyama, X. Xin, Disturbance estimation and rejection - an equivalent input disturbance estimator approach, in: 43rd IEEE Conference on Decision and Control (CDC), Vol. 2, 2004 , pp. 1736-1741. doi:10.1109/CDC.2004.1430295. 